\title{
Acute Barium Toxicity from Ingestion of "Snake" Fireworks
}

\author{
Sean H. Rhyee, MD, MPHa, Kennon Heard, $M D^{b, c}$
}

aDepartment of Emergency Medicine, Division of Medical Toxicology, University of Massachusetts Medical School, Worcester, MA

bRocky Mountain Poison and Drug Center, Denver Health

cDivision of Emergency Medicine, University of Colorado School of Medicine

\begin{abstract}
Introduction: Ingestion of fireworks has been infrequently reported in the medical literature. We describe a case of acute barium poisoning following firework ingestion.

Case Report: A 35-year-old male with a history of severe mental retardation presented with vomiting and diarrhea following ingestion of 16 small fireworks ("color snakes" and "black snakes"). His condition rapidly deteriorated and he developed obtundation, wide complex dysrhythmias, and respiratory failure. Approximately 12 hours following ingestion, his serum potassium level was $1.5 \mathrm{mmol} / \mathrm{L}$ with a serum barium level of $20,200 \mu \mathrm{g} / \mathrm{mL}$ (reference range $<200 \mu \mathrm{g} / \mathrm{L}$ ). The patient eventually recovered with ventilatory support and potassium supplementation.

Discussion: Although firework ingestion is uncommon, clinicians should be prepared for potentially severe complications. In the case of barium poisoning, treatment consists of potassium supplementation, along with respiratory and hemodynamic support.
\end{abstract}

\section{INTRODUCTION}

Fireworks are a widely-recognized source of preventable injury, particularly in children $[1,2]$. Though blast injuries and burns are commonly encountered, toxicity from ingestion of fireworks has rarely been reported. Since fireworks contain a number of hazardous substances including chlorates, nitrates, and various metals, ingestion can potentially cause significant poisoning. Barium chlorate is frequently used in fireworks and other pyrotechnics, producing a green color on ignition. While prior cases of firework ingestion have resulted in poisoning from arsenic and elemental phosphorus [3,4], barium poisoning from firework ingestion has not been previously described.

Barium poisoning causes rapid hypokalemia, resulting in cardiac dyshhythmias, muscles weakness, and respiratory failure [5-10]. Rhabdomyolysis and increased lactate levels have also been observed [5,7,8,10]. Most barium salts are well absorbed from the gastrointestinal tract; barium sulfate used in oral radiographic contrast is insoluble and therefore not absorbed. In this article, we report a case of life-threatening barium toxicity following the ingestion of consumer fireworks.

\section{CASE REPORT}

A 35 year-old male presented to a local emergency department (ED) with diarrhea and severe, repetitive vomiting. He was a longterm nursing home resident due to severe mental retardation. Medical history also included a seizure disorder, osteoporosis, and a

Keywords: fireworks, barium, hypokalemia

Notes: This study was previously presented at the ACMT CPC Competition (New Orleans, LA, October 2007).

Dr. Heard is supported in part by NIOA grant DA 020573-04.

The authors have no potential financial conflicts of interest to report.

Corresponding author: Sean Hyun Rhyee, MD, MPH, UMass Memorial Medical Center, Department of Emergency Medicine, 55 Lake Avenue North, LA-202, Worcester, MA 01655.Email: rhyees@umassmemorial.org 
prior left nephrectomy. Approximately 2 hours prior to presentation, nursing home staff witnessed the patient consume 16 small fireworks, labeled as "color snakes" and "black snakes" (Figure 1). According to staff members, he likely mistook the fireworks for chewing gum. The patient was referred to the local ED following consultation with the regional poison control center.

On arrival his vital signs were as follows: temperature $36.6^{\circ} \mathrm{C}$ $\left(97.9^{\circ} \mathrm{F}\right)$, heart rate $104 \mathrm{bpm}$, blood pressure $135 / 93 \mathrm{mmHg}$, respiratory rate $24 \mathrm{bpm}$ and a room air $\mathrm{SpO} 2$ of $97 \%$. His physical exam was unremarkable. He was combative and uncooperative with assessment, but this behavior was normal per accompanying nursing home staff. His initial lab values were notable for hypokalemia ( $2.1 \mathrm{mmol} / \mathrm{L}$ ), leukocytosis (WBC $27.2 \mathrm{k} / \mathrm{mm}^{3}$ ) and hemoconcentration (hematocrit, 55.6\%). An abdominal radiograph showed multiple, radio-opaque fragments within the stomach. The regional poison control center was consulted again about these findings. Based on the radiograph report, whole bowel irrigation with a polyethylene glycol solution was recommended. In addition, since a case of arsenic toxicity from ingestion of this type of firework [3] was reported in the POISINDEX database (Thomson Healthcare, Greenwood Village, CO), empiric treatment with dimercaprol was also advised.

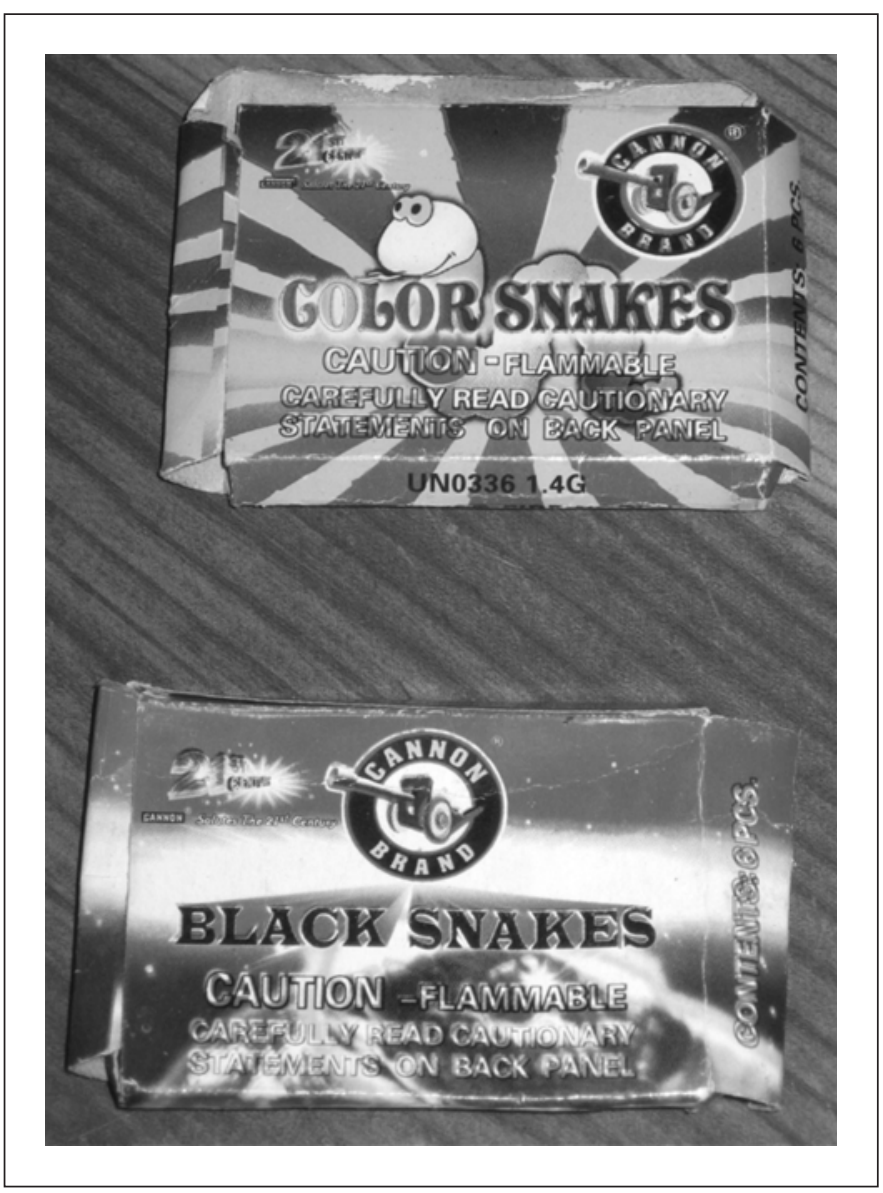

Figure 1: Packaging of ingested fireworks.
The patient did not tolerate whole bowel irrigation via a nasogastric tube due to continued vomiting. Antiemetic use was not documented. The initial hospital did not stock dimercaprol in its pharmacy, nor could any be obtained from nearby facilities. As a result, the patient was transferred to a regional tertiary referral center. While waiting for air transportation (approximately 6 hours postingestion), the patient had a 90-second episode of ventricular tachycardia associated with a blood pressure of $80 / 40 \mathrm{mmHg}$. The patient's mental status remained at baseline during this event. The ventricular tachycardia reverted to a normal sinus rhythm without intervention; blood pressure improved following a 500-mL intravenous (IV) bolus of normal saline. During air transport, another brief episode of wide complex tachycardia occurred. This episode also resolved spontaneously; documented vital signs during transport were all normal.

The patient arrived at the receiving center's intensive care unit approximately 12 hours postingestion. At this time, he was obtunded and minimally responsive to stimulation. Vital signs noted a temperature of $37.4^{\circ} \mathrm{C}$, heart rate of $117 \mathrm{bpm}$, blood pressure of $132 / 77 \mathrm{mmHg}$, and a respiratory rate of 39 breaths/minute. An arterial blood gas was notable for respiratory acidosis (pH, 7.03; pCO2, 57 mmHg; pO2, 109 mmHg; HCO3, 15 $\mathrm{mmol} / \mathrm{L}$; base excess, $16.8 \mathrm{mmol} / \mathrm{L} ; \mathrm{SaO} 2,96 \%$ on $10 \mathrm{~L} \mathrm{O} 2 / \mathrm{min}$ via a non-rebreather mask). Lab results also noted worsening hypokalemia $(1.5 \mathrm{mEq} / \mathrm{L})$. Initial serum magnesium, calcium, and phosphate levels were within the reference range. ECG on arrival recorded ventricular tachycardia (Figure 2). The patient was emergently intubated for airway protection and ventilatory support. Three IV boluses of $\mathrm{NaHCO}_{3}$ (44 mmol each) were administered empirically to treat possible sodium channel antagonist poisoning (prior to knowledge of the patient's hypokalemia). There was no immediate effect; however, within 3 hours of arrival the patient reverted to a sinus rhythm with a left bundle branch block. Blood pressure remained within the normal range during this period. An initial chest radiograph showed trace amounts of radio-opaque material in the stomach. Whole bowel irrigation was reinstituted via a nasogastric tube at 1-2 L/hour; a repeat abdominal radiograph showed no remaining radio-opaque material and irrigation was discontinued 2 hours later. A $3 \mathrm{mg} / \mathrm{kg}$ IM dose of dimercaprol was also administered on arrival; potassium repletion was also initiated. Due to the rapidly-developing hypokalemia, barium poisoning was considered. A blood heavy-metal screen, as well as tests for serum and urine barium levels, were sent following initial stabilization.

Severe hypokalemia continued during the first hospital day despite ongoing potassium supplementation. Serum potassium reached the reference range $(3.3 \mathrm{mmol} / \mathrm{L})$ approximately 16 hours after arrival; a total of $238 \mathrm{mmol} \mathrm{KCl}$ was given during this time. Potassium levels remained stable, though an additional $533 \mathrm{mmol}$ of potassium were given through hospital day 6 . In addition, 4 hours after arrival the patient developed hy- 


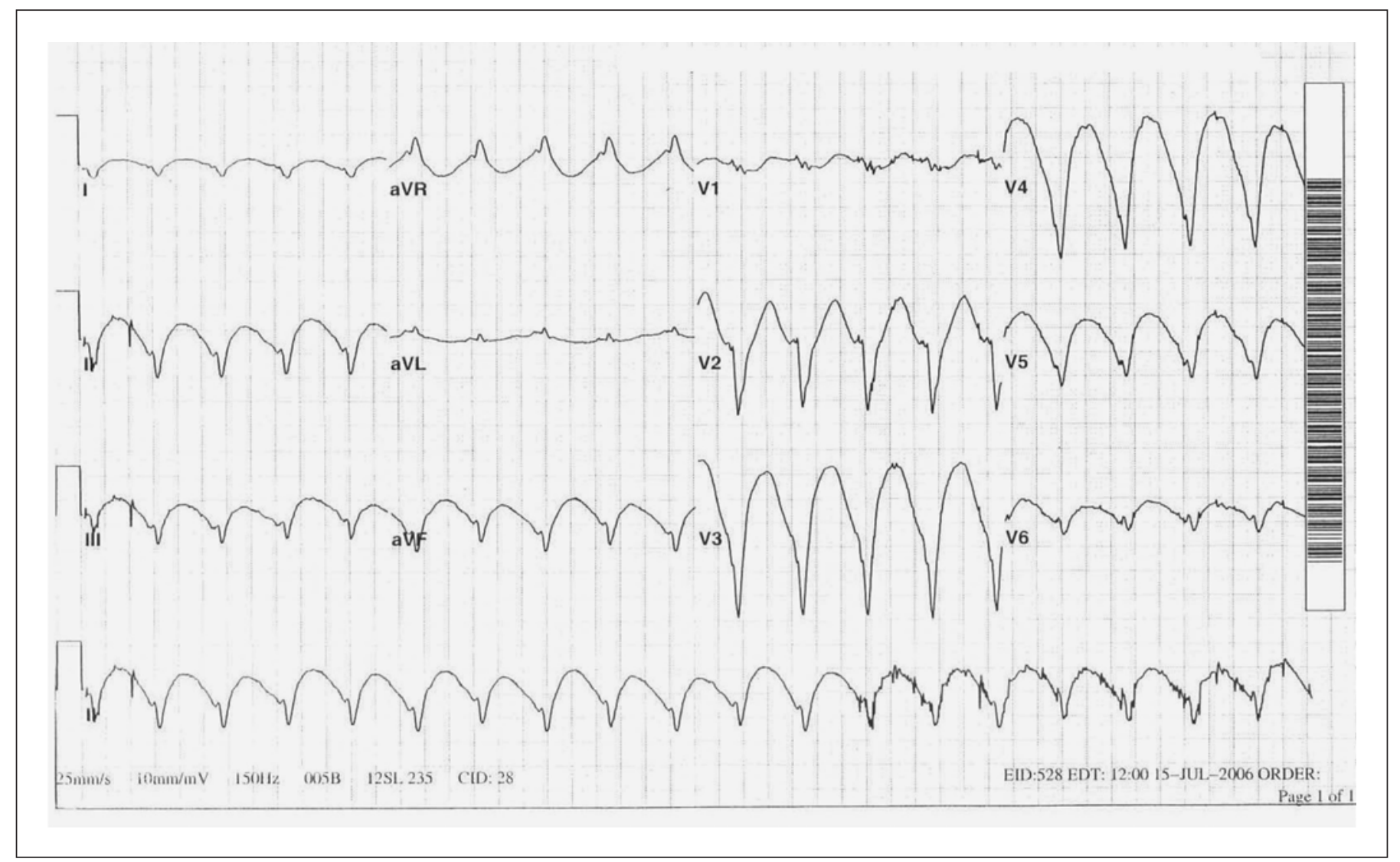

Figure 2: ECG approximately 12 hours after ingestion.

pophosphatemia $(0.22 \mathrm{mmol} / \mathrm{L})$; hypocalcemia $(1.73 \mathrm{mmol} / \mathrm{L})$ developed early during hospital day 2. Hypophosphatemia resolved within 2 days following supplementation with a total of $168 \mathrm{mmol}$ of phosphorus; hypocalcemia corrected without supplementation. Potassium doses included the potassium content of phosphorus supplements. ECG returned to normal sinus rhythm by hospital day 2 , once all electrolyte levels normalized. Interestingly, the patient had a serum troponin I level of 0.21 $\mathrm{ng} / \mathrm{mL}$ on arrival, which increased to $1.28 \mathrm{ng} / \mathrm{mL}$ within 4 hours; the reference range indicating myocardial injury was $>0.5 \mathrm{ng} / \mathrm{mL}$. Cardiology consultants attributed the rise in troponin level to the effects of the patient's electrolyte abnormalities and overall clinical condition. No cardiac stress testing or coronary angiography was performed. Repeat troponin I level was $0.05 \mathrm{ng} / \mathrm{mL}$ on hospital day 4.

His hospitalization was later complicated by aspiration pneumonia, treated with moxifloxacin and gentamicin; he was weaned off mechanical ventilation by hospital day 4 . Dimercaprol was continued at $3 \mathrm{mg} / \mathrm{kg}$ IM every 4 hours for 2 days, after which the dosing interval was extended to 6 hours. Treatment was stopped on hospital day 7 when the results of the initial heavy-metal and barium tests became available. Serum barium level was 20,200 $\mu \mathrm{g} / \mathrm{L}$ (reference range less than $200 \mu \mathrm{g} / \mathrm{L}$ ); spot urine barium level was 5,600 $\mu \mathrm{g} / \mathrm{L}$ (reference range less than 20 $\mathrm{g} / \mathrm{L}$ ). Blood levels of lead, mercury, and arsenic were within the reference range. The patient was transferred to a general medical ward on hospital day 7 , and discharged back to his nursing home on hospital day 12 .

\section{DISCUSSION}

To our knowledge, this report is the first known instance of acute barium poisoning following the ingestion of fireworks. Barium chlorate is a common ingredient in fireworks, producing a green color on ignition [10]. Despite its common usage in fireworks, previous reports of firework ingestion have not described evidence of barium toxicity; however, poisoning from other ingredients has been noted. Brayer et al. [3] reported a 3-year-old male who developed severe vomiting and diarrhea after ingesting 3 "snakes"-type fireworks. The patient recovered within 1 day with supportive care alone. A spot urine arsenic level obtained 1.5 hours after ingestion was $35 \mu \mathrm{g} / \mathrm{L}$ (within the reference range). However, analysis of the fireworks themselves noted an arsenic content of about $2 \%$ by weight. Yellow phosphorus toxicity has also been described following ingestion of South American fireworks known as diabillos [4]. Interestingly, current manufac- 
turing standards set by the American Fireworks Standards Laboratory, a fireworks industry group, prohibit the use of either arsenic or phosphorus in any fireworks products. In our case, we did not have any samples available to test the relative barium content. Multiple attempts to contact the manufacturer in Hong Kong were unsuccessful.

Our patient exhibited severe and rapid hypokalemia, the classic finding in acute barium poisoning [5-10]. The serum potassium level declined from a presumably normal level to $1.5 \mathrm{mmol} /$ L within 12 hours of ingestion. Hypokalemia produced many of the other observed clinical effects such as muscle weakness, cardiac dysrhythmias, and respiratory failure. In vitro studies note that barium inhibits potassium efflux across cell membranes [11-13], suggesting that hypokalemia results from intracellular sequestration. This hypothesis is supported by a canine study that demonstrated marked hypokalemia following $\mathrm{BaCl}_{2}$ infusion without an increase in urinary potassium secretion. The study also noted an increase in $\mathrm{RBC}$ potassium content during $\mathrm{BaCl}_{2}$ infusion [14].

While previous reports of barium toxicity have described hypophosphatemia $[7,10]$, the etiology of our patient's hypocalcemia is unclear. In previous case reports of barium poisoning, serum calcium levels were either not reported or within the reference range [5-10]. Barium is not known to affect calcium and phosphorus metabolism; in addition, the patient lacked any baseline renal or endocrinological conditions associated with hypocalcemia or hypophosphatemia.

The patient's transient elevation of troponin I is also of interest. Johnson et al. [10] reported mild elevations in CK-MB fractions (i.e., percentage of total $\mathrm{CK}$ ) following barium carbonate ingestion, but no other reports have described similar findings. Barium is not known to be directly toxic to myocardial tissue. Hypokalemia definitely provokes cardiac arrhythmias, but is not considered a primary cause of myocardial injury. The patient did have one documented episode of hypotension approximately 20 hours before the elevated troponin measurement. However, this episode was brief and unlikely to have been sufficient to cause myocardial infarction in a 35-year-old with no known cardiac disease. One possibility for the rise in troponin I could be the patient's hypophosphatemia, which developed concurrently. In vitro models have demonstrated impaired cellular energy production in myocardium subjected to phosphate depletion [15]. Myocardial injury could have resulted from this disruption of cellular metabolism.

The primary treatment for barium toxicity is correction of hypokalemia. Repletion should be carried out aggressively; however, serum potassium levels should be followed closely during treatment as rebound hyperkalemia has been reported following as little as $80 \mathrm{mmol}$ of potassium [7]. Airway and cardiopulmonary support should also be instituted as required. If ingestion of soluble barium is recognized early, oral administration of magnesium sulfate is of theoretical benefit via formation of insoluble barium sulfate [8-10]. Though our patient received $82 \mathrm{mmol}$ of magnesium (8 g MgSO4) over 5 days without apparent ill effect, IV magnesium is not recommended due to the theoretical risk of forming barium sulfate within the vasculature. Our patient also received empiric bicarbonate therapy during initial resuscitation; in known barium poisoning, bicarbonate should be avoided due to risk of worsening hypokalemia. Hemodialysis and continuous venovenous hemodialysis (CVVHD) have also been reported to be useful in rapidly eliminating barium and correcting hypokalemia $[5,8,16]$, though most cases appear to resolve with medical management.

In conclusion, this case demonstrates that life-threatening barium toxicity can result from ingestion of fireworks. We recommend that patients exposed to these products be referred to a healthcare facility for monitoring of serum potassium. If hypokalemia occurs, the clinician should be prepared to administer very large doses of potassium if necessary.

\section{REFERENCES}

1. Witsaman RJ, Comstock RD, Smith GA. Pediatric fireworks-related injuries in the United States: 1990-2003. Pediatrics. 2006;118:296-303.

2. Centers for Disease Control and Prevention. Injuries from fireworks in the United States. MMWR. 2000;49:545-546.

3. Brayer AF, Callahan CM, Wax PM. Acute arsenic poisoning from ingestion of "snakes." Pediatr Emerg Care. 1997;13: 394-396.

4. Tafur AJ, Zapatier JA, Idrovo LA, et al. Bone marrow toxicity after yellow phosphorus ingestion. Emerg Med J. 2004;21:259-260.

5. Koch M, Appoloni O, Haufroid V, et al. Acute barium intoxication and hemodiafiltration. J Toxicol/Clin Toxicol. 2003;41: 363-367.

6. Jacobs IA, Taddeo J, Kelly K, et al. Poisoning as a result of barium styphnate explosion. Am J Ind Med. 2002;41: 285-288.

7. Sigue G, Gamble L, Pelitere M, et al. From profound hypokalemia to life-threatening hyperkalemia. Arch Intern Med. 2000;160:548-551.

8. Schorn TF, Olbricht C, Schuler A, et al. Barium carbonate intoxication. Intensive Care Med. 1991;17:60-62.

9. Downs JC, Milling D, Nichols Clay A. Suicidal ingestion of barium-sulfide-containing shaving powder. Am J Forensic Med Pathol. 1995;16:56-61.

10. Johnson $\mathrm{CH}$, VanTassell VJ. Acute barium poisoning with respiratory failure and rhabdomyolysis. Ann Emerg Med. 1991;20:1138-1142.

11. Sperelakis N, Schneider MF, Harris EJ. Decreased $\mathrm{K}^{+}$ conductance produced by $\mathrm{Ba}^{++}$in frog sartorius fibers. J Gen Physiol. 1967;50:1565-1583.

12. Volle RL. Blockade by barium of potassium fluxes in frog sartorius muscle. Life Sci. 1970;9:175-180. 
13. Nagel W. Inhibition of potassium conductance by barium in frog skin epithelium. Biochem Biophys Acta. 1979;552: 346-357.

14. Roza O, Berman LB. The pathophysiology of barium: hypokalemic and cardiovascular effects. J Pharmacol Exp Ther. 1971;177:433-439.
15. Brautbar N, Altura BM. Hypophosphatemia and hypomagnesemia result in cardiovascular dysfunction: theoretical basis for alcohol-induced cellular injury. Alcohol Clin Exp Res. 1987;11:118-126.

16. Wells JA, Wood KE. Acute barium poisoning treated with hemodialysis. Am J Emerg Med. 2001;19:175-177. 